\title{
Simulating electrostatic effects on electronic transitions in proteins
}

\section{Author: Patrik R. Callis}

This is an Accepted Manuscript of an article published in Molecular Simulation in January 2015, available online: http://www.tandfonline.com/10.1080/08927022.2014.923571.

Callis, Patrik R.. Simulating electrostatic effects on electronic transitions in proteins. Molecular Simulation. June 2014. Pages 190-204.

https://dx.doi.org/10.1080/08927022.2014.923571

Made available through Montana State University's $\underline{\text { ScholarWorks }}$

scholarworks. montana.edu 


\section{Simulating Electrostatic Effects on Electronic Transitions in Proteins}

Patrik R. Callis

Department of Chemistry and Biochemistry

Montana State University

Bozeman, MT 59717-3400

Author Information:

Patrik R. Callis

Department of Chemistry and Biochemistry

PO Box 173400

Montana State University

Bozeman, MT 59717-3400

Email: pcallis@montana.edu

Phone: (406) 994-5414

Fax: (406) 994-5407 


\begin{abstract}
Biopolymer fluorescence in biology and biochemistry is increasingly used for characterizing equilibrium, dynamics, and imaging. This is typically done by monitoring wavelength and intensity changes without necessarily knowing what causes such changes in detail. Simulations have been at the core of the considerable recent progress in improving the microscopic understanding of wavelength and quenching of fluorescence intensity in biopolymers. This review focuses on one of the most used intrinsic probes for protein behavior, tryptophan (Trp), which is arguably now one of the best understood probes of internal structure and dynamics for proteins - despite its reputation to the contrary. In this review, we highlight selected classical molecular dynamics in combination with quantum mechanics (MD + QM) simulations from our group and others during the past 20 years that support this view. The work includes simulations of time dependent wavelength shifts in solvents and proteins, fluorescence quenching rates, dielectric compensation by water, heterogeneity of quenching rates, and applications to protein folding.
\end{abstract}

Keywords: QM-MM simulations; proteins; tryptophan; fluorescence; electron transfer 


\section{Introduction}

Molecular simulations serve a diverse population of scientists and engineers, often as a means to help develop, and sometimes discover, the underlying theories to explain surprising experimental results. Many intriguing phenomena associated with absorption and emission of light from proteins continue to present themselves during investigations involving electronic spectra, and these inevitably breed a desire to understand the underlying physical principles. Our entry into simulations grew from a combination of experimental and quantum mechanical studies of the electronic structure of the indole molecule and its derivatives related to the amino acid tryptophan (Trp), whose structures are part of Figure 1. Trp is one of the most widely exploited natural chromophores for two reasons. One is that proteins - the machines of life-are so widely studied, and the other is because Trp fluorescence intensity and peak wavelength $\left(\lambda_{\max }\right)$ are so sensitive to the precise details of the surrounding protein and solvent structure that for almost any functional change a protein does, Trp fluorescence can monitor the extent of that change.
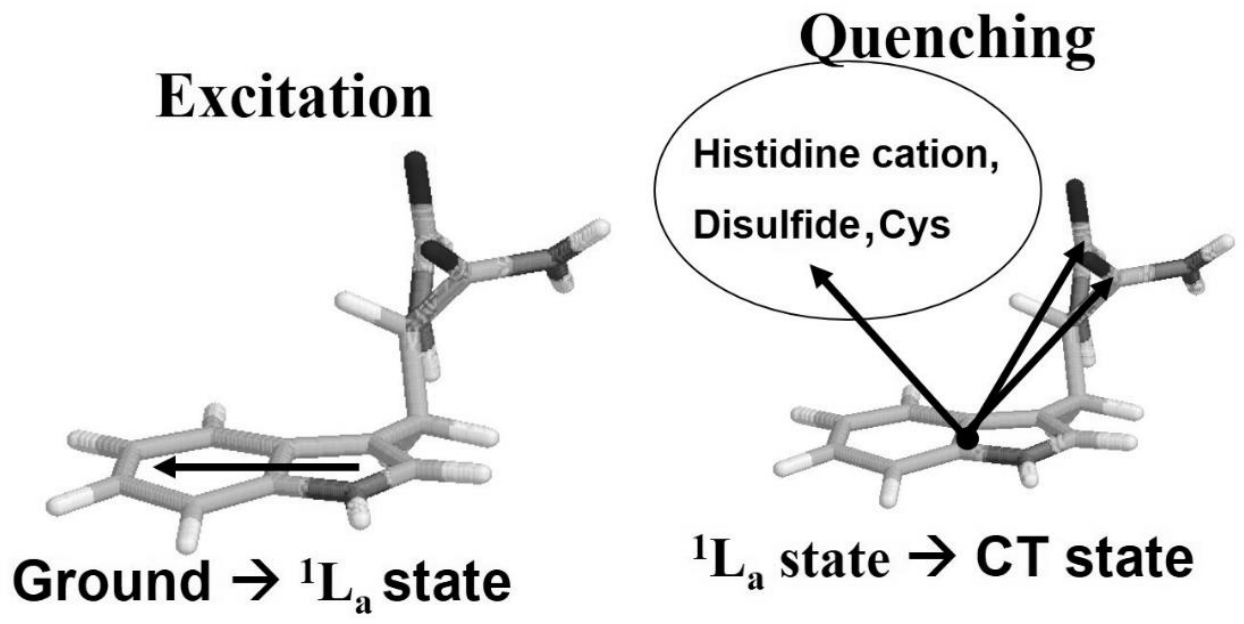

Figure 1. Tryptophan as connected to protein backbone amides, with electron density changes indicated for excitation and quenching by ET. The local electric potential difference (voltage and field strength and direction) determine whether the fluorescence will be red or blue shifted and whether an electron acceptor will or will not quench the fluorescence. Color coding: $\mathrm{C}=$ light gray; $\mathrm{N}=$ dark gray; $\mathrm{O}=$ black; and $\mathrm{H}=$ white. Attachment to the backbone is at the 3-position of the indole aromatic ring. A common mode of fluorescence quenching is by ET from indole ring to one of the amide groups, or to the histidine cation, disulfide, or cysteine side chains. The basic chromophore of tryptophan without amides is 3-methylindole (3MI).

Understanding electronic spectroscopy in disordered, ambient temperature, condensed phase, however, is challenging because of the constantly fluctuating intermolecular interactions with closely neighboring molecules. One of the most compelling reasons for simulations is that most of the important action happens during fluctuations (e.g., energy barrier crossing). This important fact remains essentially invisible to those who do not study simulations. A relatively small number of groups have approached this problem by combining classical molecular dynamics (MD) simulations with quantum mechanical QM computations. The latter typically is performed on a relatively small part of the protein that absorbs and emits light (the 
chromophore), but with input from the MD part about the electrostatic interactions that perturb the chromophore. This approach has seen a range of strategies. At one extreme only a small amount of the protein/solvent surroundings is included, but with relatively high level QM applied to the chromophore during a relatively short trajectory.[1-5] At the other extreme, virtually all of the surroundings (all of the protein and several thousand explicit water molecules) is included during long trajectories, but at the expense of using semiempirical QM, or perhaps only Coulomb's law.[6-14] The work from our laboratory falls mostly into the latter category, driven by our conviction that the greater sampling rate possible is well worth the minor deficiencies in most semiempirical methods. We highlight evidence for this position here by presenting a montage that captures some of the novel views uncovered by our efforts.

Arieh Warshel, one of this year's Nobel laureates for pioneering simulations of protein energetics and dynamics, 22 years ago wrote that the underlying basis for the powerful catalytic ability of enzymes (proteins) lies in electrostatics.[15] Most physical chemists realize that all of chemistry - and therefore biology, geology, material science, etc. - depends on electrostatics at the most fundamental level. Yet this fact and Warshel's message are not as fully appreciated as one would expect.[16] The importance of electrostatics is ever in the foreground of the simulations presented here, so much so that an appropriate alternate title for this article could be "Exploring the electrostatic landscape of proteins with tryptophan fluorescence."[17]. We have presented related reviews in recent years.[18-22]

\section{Simulations led to the discovery of femtosecond inertial response of $\mathrm{H}_{2} \mathrm{O}$}

Few would deny that water energetics and dynamics is of fundamental importance in the functioning of biopolymers. Indeed, the importance of water in slightly surprising roles is a major theme for this review, as witnessed by the mention of the word water 60 times. It is fitting, therefore that molecular simulations claimed a major scientific milestone when Maroncelli and Fleming used computer simulations to model the relaxation times of liquid water in response to suddenly produced charge changes, caused by absorption of a photon of light for example. [23] [24]. Their results were seminal in drawing attention to the importance of extremely fast "inertial" response to sudden charge distribution changes - responses that had been completely missed by diligent efforts at that time to describe solvation relaxation times from dielectric dispersion data. [25] (This behavior was actually noted much earlier by Rao and Berne[26], but went largely unnoticed because the predictions could not begin to be verified experimentally then.) Experimental verification of inertial relaxation was done in Fleming's lab[27], and we might say that the motivation to obtain these experimental results was also the motivation to do the simulations.

The core of this section is to discuss two very simple - but particularly revealingsimulations of exactly what causes such astonishingly fast behavior, so fast that it was not anticipated from theoretical or experimental means. Maroncelli's description[24] certainly makes clear why this was not anticipated. He notes that the motions involved in the solvent response are mainly reorientational motions that are completely independent of the intermolecular forces between the solvent molecules. Second, these motions are rapid, not 


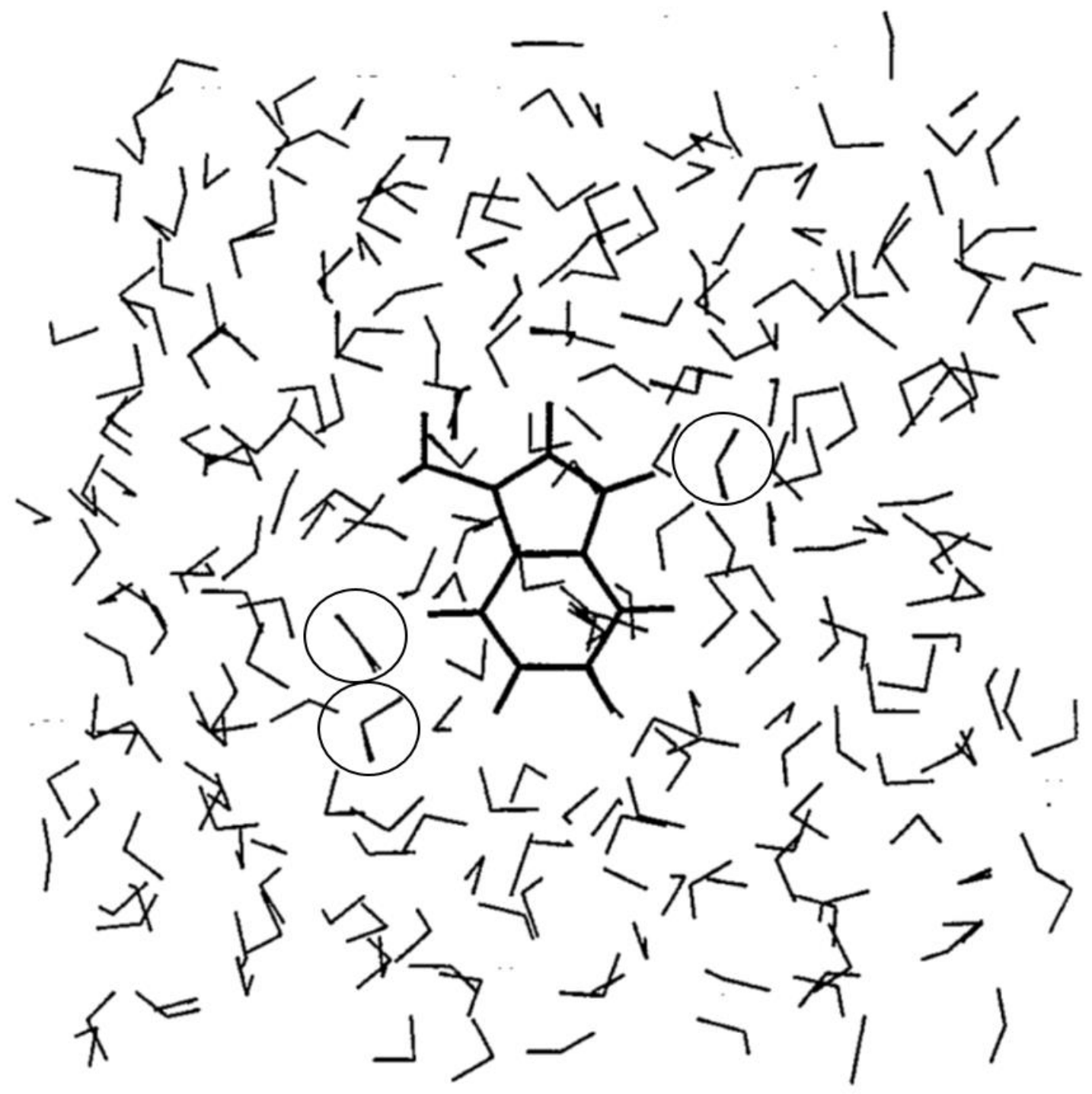

Figure 2. Superimposed snapshots of two configurations at simulation time, $t=20 f s$ for fixed 3-MI in TIP3P water. At $t=0$, both configurations were identical. One of those two was excited to the ${ }^{1} L_{a}$ state, while the other was not. The difference between the two configurations at $t=20 \mathrm{fs}$ corresponds to the effect on the solvent of solute excitation. There are no discernible translational differences and only a few barely discernible rotational differences, such as those in circles. In this example of inertial response, all of the small changes are in response to torque from the excited dipole, and therefore all are contributing to $50 \%$ of the total stabilization after only 20 fs.

because of dynamical intermolecular interactions, but because of their small amplitude, which nevertheless in sum create large stabilization of the newly created dipole. He illustrated that point with a highly creative simulation, one that further exposed the nature of the fast dynamics and left no doubt that this was the correct description. A series of "rigid-cage" simulations were performed, wherein all solvent molecules but one are "frozen" at some initial configuration selected from an equilibrium ground state ensemble. The one remaining solvent molecule undergoes dynamics in the rigid environment formed by its neighbors. Note that this is not 
frozen in the sense of low temperature. It is a fictitious "thought experiment" in which the random positioning of the solvent about the solute is consistent with room temperature, yet all but one of the molecules is forbidden to move (something only possible in a computer simulation). Surprisingly, there is space for the single free water molecule in each case to move sufficiently in response to the sudden change in solute charges. The sum of the stabilizations from the individual molecules was very close to that given when all were free to respond simultaneously.

My group and I broadened our investigations from purely electronic structure experiments and theory aimed at understanding the fundamentals of the enigmatic Trp into the arena of MD simulations during a sabbatical year in Bruce Hudson's laboratory. (Initially, our focus was on gaining a better grasp of the lowest absorption band which had contributions from two distinct electronic states, termed ${ }^{1} \mathrm{~L}_{\mathrm{a}}$ and $\left.{ }^{1} \mathrm{~L}_{b}\right)[18,28]$ Although it was clear that the more polar ${ }^{1} \mathrm{~L}_{\mathrm{a}}$ would be the emitter of the most red-shifted fluorescence, there was little evidence concerning whether ${ }^{1} \mathrm{~L}_{\mathrm{a}}$ or the less polar ${ }^{1} \mathrm{~L}_{\mathrm{b}}$ emitted at short wavelengths. At that time in the late 1980 s there was also interest in whether MD methods could predict fluorescence $\lambda_{\max }$ and how fast water would relax around the excited state, as an extension of the simulation of absorption spectra by Ilich and Prendergast.[29] With the help of Dan Harris, we carried out MD + QM simulations of the fluorescence of 3-methylindole (3MI) in water and the time dependence of $\lambda_{\max }$ from the ${ }^{1} \mathrm{~L}_{\mathrm{a}}$ state.[30,31] (3MI is better than indole as a model for Trp in proteins because the methyl group at the 3-position shifts the ${ }^{1} \mathrm{~L}_{a}$ state to lower energies relative to the ${ }^{1} \mathrm{~L}_{b}$ state, mimicking the effect of the alanyl group, which connects the indole ring to the protein backbone, as seen in Figure 1).

From that work, we offered a similarly revealing view, complementary to that of Maroncelli, of the early time dynamics in the inertial response of water to excitation of 3MI. Figure 2 shows two frames of 252 water molecules from two trajectories originating from identical configurations of equilibrated ground state. For one of these, the indole ring is excited (the charges are instantly changed to those of the ${ }^{1} \mathrm{~L}_{\mathrm{a}}$ state) and propagated with these charges; the other trajectory continues with the ground state charges. The two final configurations are shown superimposed after only 20 fs of propagation, during which time about $50 \%$ of the total energy relaxation has occurred $\left(-2300 \mathrm{~cm}^{-1}\right)$. The bulk of the response in the first $20 \mathrm{fs}$ is seen to be due to small differential rotations (barely discernable and best seen for the circled cases in the figure) mostly by about 10 water molecules within 4-6 $\AA$ of the solute. These are special molecules that happen to be oriented so as to feel a strong instantaneous change in torque due to the new charge distribution. The largest rotation difference was $20^{\circ}$, although the average thermal rotation amplitude during $20 \mathrm{fs}$ was $35^{\circ}$. The signature of this inertial response is that each of these special molecules whose trajectory made small angular deviations from the unperturbed trajectory contributed a lowering of the energy. This is why so few molecules lower the energy so effectively. There is no diffusive component, and each properly oriented water heads downhill in energy. The reason for the fast response lies, as pointed out by Maroncelli, in the smallness of the deviations. During this short time, translational motion of the center of masses is negligible (always less than $0.005 \AA$, and in most cases, less than $0.001 \AA$ ). 


\section{Wavelength $\left(\lambda_{\max }\right)$ predictions in proteins}

Serendipity is more the rule than the exception as a normal flow of science. Ten years earlier during a sabbatical year in A. C. Albrecht's laboratory, we gained two-photon absorption (TPA) expertise, and also an appreciation for the effectiveness of Zerner's INDO/S-CIS (Zindo),[32-34] a spectroscopically calibrated version of an approximate Hartree Fock method, INDO, developed by Pople and co-workers.[35] We subsequently used Zindo in our MD simulations of 3MI in water.[31] We gained much more respect for Zindo after Robert Woody asked us to compute transition dipoles of DNA bases in crystals from the fields and potentials he had computed. Zindo results in vacuum were being maligned for poor agreement with the crystal experiments, but provided reasonable agreement when the strong crystal fields were included. [36,37]

Even more confidence in Zindo emerged from our prediction - before the experiments - that TPA, using circular and linearly polarized light could distinguish ${ }^{1} \mathrm{~L}_{\mathrm{a}}$ from ${ }^{1} \mathrm{~L}_{\mathrm{b}}$. Subsequent cryogenic experiments on indole derivatives provided a detailed picture of the type of electrostatic environment that would cause ${ }^{1} \mathrm{~L}_{\mathrm{a}}$ to be the emitting state.[18,38-41] It was clear that ${ }^{1} \mathrm{~L}_{\mathrm{a}}$ would be the emitting state in virtually all proteins.[18]

This knowledge provided the confidence to start simulations of Trp fluorescence on proteins. We began learning about proteins and how to interface our QM calculations with MD simulations of proteins surrounded by explicit water. Along the way, we were encouraged by the studies by Burstein and co-workers [42-44], and by early simulations by Marchi et al. [8,9]

In addition to the ${ }^{1} \mathrm{~L}_{\mathrm{a}}$ nature of the emitting state, there emerged another crucial piece of knowledge that helped spur us into simulations of $\operatorname{Trp}$ fluorescence $\lambda_{\max }$ values in proteins: without exception, a large number of quantum chemical computations, including our own, on indole and 3MI, had predicted that electron density is shifted from the pyrrole ring to the benzene ring upon excitation to the ${ }^{1} \mathrm{~L}_{\mathrm{a}}$ state. See reference[18] for a review. Therefore, positively charged residues near the pyrrole end or negative charges near the benzene end of the Trp ring would be expected to shift $\lambda_{\max }$ to shorter wavelengths (produce a "blue" shift), with the opposite configuration producing a "red" shift.(Figure 1)

For modelling the steady state $\lambda_{\max }$ in proteins, we continued to use INDO/S-CIS (Zindo). We were focused only on the electrostatic effect of the protein and solvent on the fluorescence wavelength. To avoid distractions from the harmonically vibrating bond lengths, which add a noisy background to the effect of the electric field, all calculations were performed on $3 \mathrm{MI}$ in a standard geometry and orientation, but with the electric potentials on the atoms given by the instantaneous values from the MD. This was also to facilitate identification of the ${ }^{1} \mathrm{~L}_{\mathrm{a}}$ and ${ }^{1} \mathrm{~L}_{\mathrm{a}}$ states, which sometimes became mixed or changed energy order. Rotating the coordinates of the indole has no effect on the atom potentials (scalars), but the field vectors must be rotated with the molecule. This turns out to be effectively a pro forma exercise, however, because the fields have little effect on the predicted wavelength. The field atomic matrix elements affected are largely localized to single atoms.[36] The fields and potentials came from the point charges of each atom of the non-QM protein and all waters as given by the Charmm22 all-atom force field, always with a dielectric constant of unity. The indole ring was given the Zindo Löwdin charges. The ground state geometry came from a crystal structure.[45] The difference between ground and excited state geometries was given by a (CIS-HF/3-21G) ab initio calculation, which gives 
accurate bond lengths despite the modest basis set. 30-ps trajectories were used in that early study. The protein along with crystallographic waters and metal ions were all solvated by a $30 \AA$ sphere of explicit TIP3P water. In this study, and in several that followed, QM charges on the indole ring were fed back to the MD every $10 \mathrm{fs}$ (10 steps), thereby introducing a realistic polarizability, without which agreement with experiment would have been poor.

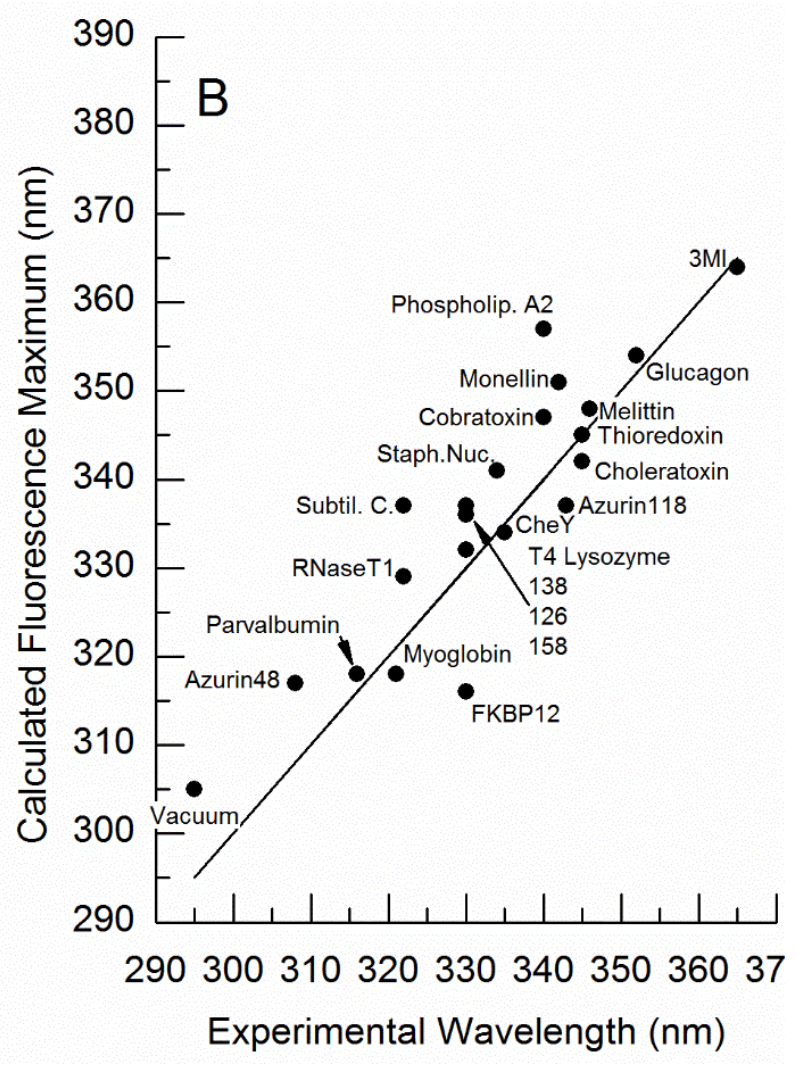

Figure 3. Plots of calculated versus experimental fluorescence maximum wavelengths for 19 Trps in 16 proteins and for 3-MI in water. Charges on the Trp ring are multiplied by 0.80 to improve the fit, and the calculated values are averages over the 2400 values calculated during the last 24 ps of $30 \mathrm{ps}$ trajectories.

Figure 3 shows the results for predictions of $\lambda_{\max }$ for 19 Trps in 16 proteins,[10] starting with X-ray structures, and including only electrostatic interactions of the Trp ring electron density with all other atoms in the simulation (with a dielectric constant of 1). Because we found $\lambda_{\max }$ for $3 \mathrm{MI}$ by itself in water predicted to be $390 \mathrm{~nm}$ instead of the observed $367 \mathrm{~nm}$, we reduced the Zindo charges on the ring (as seen by the MD environment) by $20 \%$. With this one adjustable parameter, the mean absolute deviation between the predicted and observed $\lambda_{\max }$ was $6 \mathrm{~nm}$. 
A number of new details regarding the microscopic determinants of wavelength shifts emerged from this study:

1) The information that the ground $\rightarrow^{1} \mathrm{~L}_{\mathrm{a}}$ excitation transfers electron density from the pyrrole ring to the benzene ring combined with the knowledge that $\lambda_{\max }$ is determined primarily by the electric potential difference across the long axis of the indole ring was strongly solidified. This means that the relative direction of a charge from the Trp ring is crucial: positive charges create a red shift when near the benzene ring end and a blue shift when near the pyrrole ring end, with the size of the shift being inversely proportional to the distance from the center of the Trp ring (at the long distance limit). The reverse is true for negative charges.

2) Both water and protein show a diversity of ratios in their contributions to $\lambda_{\max }$, but the ratio is generally difficult to anticipate. When charged groups lie close to Trp, they usually dominate the mechanism of the shift, but the explicit water was almost always found to exhibit long-range ordering by the charges that opposed the large direct effect by the charges. Sometimes water even contributed a blue shift.

3) Water exposure does not always cause a large red shift. If the exposure is only along one edge of the Trp, only modest red shifts from water are possible. If, however, one or both faces of the indole ring are water-exposed, the wavelength of the fluorescence peak is usually near the maximum seen for water-exposed Trp in proteins $(\sim 350 \mathrm{~nm})$. Often in such cases a few water molecules make especially large red shifts $(\sim 20 \mathrm{~nm})$ due to exciplex-like H-bonds with negatively charged $\mathrm{C}$ atoms of the benzene ring in the ${ }^{1} \mathrm{~L}_{\mathrm{a}}$ excited state.

4) Some buried Trps were seen to receive sizeable $(10-20 \mathrm{~nm})$ red shifts from water. This appears to be due to the collective action of regions of water up to $25 \mathrm{~nm}$ distant that are probably oriented by the charges and/or shape of the protein.

5) In light of the results of this study, the absence of experimental blue shifts beyond $308 \mathrm{~nm}$ seemed remarkable. For Trp 48 of azurin, the blue shift results from the Trp being deeply buried in a hydrophobic pocket, with all water molecules at $>10 \AA$ distant. We believe that the paucity of larger blue shifts, which are plausible in the context of this study, is because the ground and excited state dipoles point in roughly the same direction. Charges positioned to create an extremely blue-shifted fluorescence would necessarily destabilize the protein ground state. This may have been a factor in the evolution of proteins. (Epilogue: Broos et al. have confirmed that even the $308 \mathrm{~nm}$ fluorescence of $\operatorname{Trp} 48$ of azurin emits entirely from ${ }^{1} \mathrm{~L}_{\mathrm{a}}$, [46]. More recently, Trp72 of the M97V mutant of transhydrogenase has been reported to emit partly from the ${ }^{1} \mathrm{~L}_{\mathrm{b}}$ state, with $\lambda_{\max }$ of $\left.306 \mathrm{~nm} .[47]\right)$

6) This method is general, and can easily be applied to other chromophores embedded in similar media, for example flavins in proteins.[48]

7) This work gave evidence that the CHARMM22 force field provides an accurate description of local electric fields in proteins, and complements work aimed at predicting $\mathrm{pKa}$ values and redox potentials, which are also sensitive to electric potential differences. 


\section{Fluorescence quenching predictions in proteins}

Fluorescence quenching refers to a process by which the excitation energy of an individual fluorescing molecule interacts with another molecule, ion, or atom (the quencher) in such a way that the fluorophore excited state population is rapidly reduced, greatly reducing the probability of fluorescence. The excitation energy may be entirely transferred to the quencher, or the mixing of fluorophore and quencher wavefunctions may facilitate fast nonradiative decay within the fluorophore. The quencher typically does not fluoresce significantly, or does so at a different wavelength. Quenching, therefore, is at the heart of the use of fluorescent molecules as sensors for molecular events that modulate intensity.

We chose to pass up a grand opportunity to delve immediately into studies of time dependent fluorescence Stokes shift (TDFSS, i.e., shifting of $\lambda_{\max }$ following sudden excitation) of Trp in proteins. Instead, we turned to what was, to us, a looming, irresistible question: the long-standing question as to the origin of Trp fluorescence intensity (quantum yield) variability in proteins - a puzzle because the quantum yield of 3MI is barely affected by solvent[49] when the indole ring is not in a protein. Despite five decades of reporting fluorescence yields and lifetimes of Trp fluorescence from single-Trp proteins, even with good crystal structure information, no one could predict or understand in a fundamental way why some Trps fluoresced as strongly as if not in a protein while others would fluoresce up to 30-fold more weakly.

The way forward was framed with two well-established facts: 1)the quantum yield of 3MI was almost independent of solvent polarity[49] and 2)by attaching two amide groups to $3 \mathrm{MI}$ in a manner found for all Trps in proteins (as shown in Figure 1), the quantum yield was reduced by $50 \%$ in water.[50] In addition, there were several papers that, taken together, made an extremely good case that the quenching process was due to electron transfer (ET) from the excited indole ring to one of the two nearby backbone amides.[51-53] What remained was to understand which variable(s) conspired to allow this EY to occur in some cases and not in others.

At some point, we realized that a charge transfer (CT) state arising from an intramolecular ET is just another excited state of the molecule, and could be treated in a manner closely related to how we treated the wavelength predictions. We simply extended the QM chromophore to include the two closest amides, and examined the energy of those excited states that had the characteristic electron density signature of a CT state in which an electron was transferred from the indole ring to one of the amides. To do this, a modification of the oxygen parameters in Zindo was required, which fortunately had already been put in place by Li et al. [54]

Figure 4 shows four examples that serve to summarize the state of this particular "art". In each panel, the lower curve (points) is the energy of the fluorescing state $\left({ }^{1} \mathrm{~L}_{\mathrm{a}}\right)$, and the solvent is in equilibrium with this state. The upper curve (lines) is the energy of the CT state in the solvent reaction field created by the ${ }^{1} \mathrm{La}$ charges. (The energy units are in $10^{3} \mathrm{~cm}^{-1}=0.125 \mathrm{eV}=12$ $\mathrm{kJ} / \mathrm{mol}$.) The upper panels (A and B) are for two representative proteins that exhibit low fluorescence, while the panels $\mathrm{C}$ and $\mathrm{D}$ are representative of highly fluorescent proteins. Note the correlation of the ${ }^{1} \mathrm{~L}_{\mathrm{a}}-\mathrm{CT}$ energy gap with quantum yield. ET cannot happen if the zero point of the ${ }^{1} \mathrm{~L}_{\mathrm{a}}$ state lies below that of the CT state. 
Why ET quenches fluorescence is revealed by the simulation of an actual "quenching event" in each panel of Figure 4. These are marked by the abrupt drop in energy of the CT state near the end of each trajectory. These are brought about by instantly changing the charges on the chromophore atoms, at an arbitrary point in time, to those characteristic of the CT state, i.e., the indole ring takes on a delocalized positive charge, and one of the nearest amides becomes
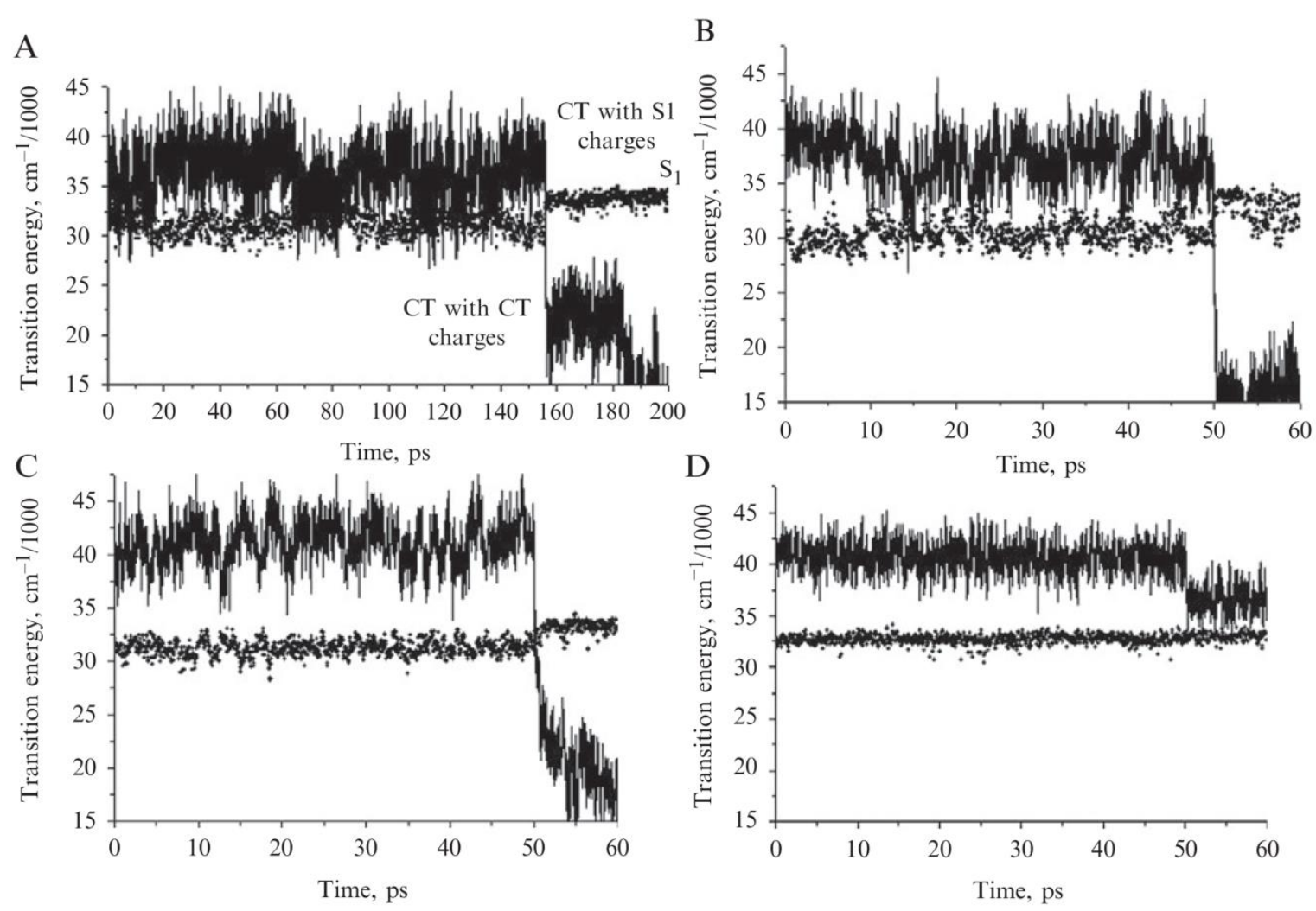

Figure 4. Typical QM-MD trajectories of ${ }^{1} \mathrm{~L}_{\mathrm{a}}$ (points) and CT (solid lines) state energies from Zindo. A) For Trp 126 Dsba (weakly fluorescent); B) phospholipaseA2 (weakly fluorescent); C) Trp140 in Staphylococcal nuclease (strongly fluorescent); D) Trp48 in apo-azurin (strongly fluorescent, extremely blue shifted, and very narrow band width). In each panel, during the early part of the trajectory the Trp charge distribution is that of the ${ }^{1} \mathrm{~L}_{\mathrm{a}}$ (fluorescing state). The sharp drop at the later end is the response of the system due to changing the charge distribution to that of the CT state at an arbitrarily chosen time point, as if an ET to the amide had taken place. The sharp drop is caused by the classical inertial response by the environment (mainly water) through the MD to the new charge distribution.

negative. The large, steep part of the drop in CT energy happens in $100 \mathrm{fs}$, and is an example of the important role of inertial response introduced in Section 2 above. Except for the case of azurin (Figure 4(d)), where the Trp is in a completely non-polar environment, the CT state becomes irreversibly trapped far below the fluorescing state and remains there. This is quenching, because we presume in nature that the system will return to the ground state more quickly than returning to high enough energy to regain the ${ }^{1} L_{a}$ state. 
In the curves of Figure 4, we used a time-dependent quantum mechanical framework, $[55,56]$ which has been to be shown to be equivalent to the well-known Marcus formulation of ET theory.[57] The preliminary computations suggested to us that coupling of the CT and ${ }^{1} \mathrm{La}_{\mathrm{a}}$ states was weak enough to justify the use of the Fermi Golden Rule, whose general form for the ET rate constant is given by:

$$
k_{\mathrm{et}}(t)=\frac{2 \pi}{\hbar} V_{e t}^{2}(t) \rho\left(\Delta E_{00}(t)\right)=4 \pi^{2} c V_{e t, w n}^{2}(t) \rho\left(\Delta E_{00, w n}(t)\right),
$$

where $V_{e l}$ is the Hamiltonian matrix element between the ${ }^{1} \mathrm{~L}_{\mathrm{a}}$ and CT states and $\rho$ is the density of final states (states per unit energy), which is a function of the difference of zero-point energies of the two states, $\Delta \mathrm{E}_{00}$. In a protein/solvent environment, both $V_{e l}$ and $\rho$ are functions of time, as is easily seen in the trajectories of Figure 4. The right hand version of equation (1) results from using $\mathrm{cm}^{-1}$ for all energy units and $\mathrm{c}$ is the speed of light in $\mathrm{cm} \mathrm{s}^{-1}$.

Our first application[11,58] used the approximate form:

$$
k_{\mathrm{et}}(t)=4 \pi^{2} c V_{e t, w n}^{2}\left\langle\rho\left(\Delta E_{v e r t, w n}+D_{v e r t, w n}\right)\right\rangle_{t},
$$

in which $V_{e l}$ was assumed to be $10 \mathrm{~cm}^{-1}$ for all proteins. $\Delta \mathrm{E}_{\text {vert }}$ is the vertical Zindo energy difference between the lowest $\mathrm{CT}$ and ${ }^{1} \mathrm{~L}_{\mathrm{a}}$ states at the ${ }^{1} \mathrm{~L}_{\mathrm{a}}$ geometry. $\mathrm{D}_{\text {vert }}$ is formally the constant added to $\Delta \mathrm{E}_{\text {vert }}$ so that $\Delta \mathrm{E}_{\text {vert }}+\mathrm{D}_{\text {vert }}=\Delta \mathrm{E}_{00}$. In practice, $\mathrm{D}_{\text {vert }}$ was treated as an empirical fitting parameter applied to all proteins. The density of states was taken as the time average of the overlap integral of the broadened spectrum of Franck-Condon factors for removing an electron from ${ }^{1} \mathrm{~L}_{\mathrm{a}}$ of indole and that for placing it in the lowest empty orbital on formamide. The overlap depends critically on the energy gap. The procedure for constructing these curves follows what we did for predicting vibronic spectra of cold indole vapor.[59] This procedure was applied to 24 Trps in 17 proteins $[11,58]$ thereby providing the first quantitative predictions of the full 30-fold range of Trp fluorescence quantum yields (and lifetimes) observed in proteins. This was found primarily to be due to different rates of ET from the excited indole ring to the empty anti-bonding $\mathrm{MO}$ of one of two nearest backbone amides. Importantly, because a constant, empirical $V_{e l}$ was used, the predicted variation was seen to be entirely due to the variation in ${ }^{1} L_{a}-C T$ energy gap, which comes entirely from the atomic charges of all protein and water atoms assigned by the Charmm 22 force field. This array of partial charges determines the average local electric potential difference between the Trp ring and acceptor amide, and the amplitude of these potential difference fluctuations is caused by protein and solvent motions. Quite importantly, it is strongly influenced by the location of nearby charges relative to the transfer direction. A negative charge near the indole ring and/or a positive charge near the electron acceptor, will increase the ET rate (decrease $\Phi_{\mathrm{f}}$ ), because these arrangements stabilize the CT state. In the opposite case, there will be minimal ET, and the Trp will be highly fluorescent.

Although this formulation gave a reasonable fit between calculation and experiment, the ad hoc choice of only $10 \mathrm{~cm}^{-1}$ for the ET matrix element required a high density of states, which could only be achieved with $D_{\text {vert }}=-4000 \mathrm{~cm}^{-1}$ (subtracting 4000 from the computed vertical CT energy). This was a physically unreasonable empirical offset to the energy gap; the opposite was 
expected because the simulations were run with the geometry fixed at that of the CT state, thereby stabilizing the CT state relative to the ${ }^{1} \mathrm{~L}_{\mathrm{a}}$ state.

Later we made reasonable ab initio calculations of $\mathrm{Vel}(\mathrm{t})$ over short trajectories for each of the proteins in the test set.[60] The resulting ab initio matrix elements ranged from 100 to 1000 $\mathrm{cm}^{-1}$, therefore requiring smaller, more reasonable $\rho$ values for agreement with experiment. This was achieved by adding $4700 \mathrm{~cm}^{-1}$ to the calculated energy gap, a number that is consistent with running the trajectory with geometry of the $\mathrm{CT}$ state.

One might well raise the question of whether such large interactions would push the ET transfer rate out of the region of validity for the Fermi rule (non-adiabatic) into the adiabatic realm, wherein $\mathrm{k}_{\text {et }}$ becomes independent of $V_{e l}$. Certainly by the original Landau-Zener criterion, $V_{e l}$ values of these magnitudes indicate the adiabatic limit. That simple model, however, is based on two isolated levels crossing at a uniform velocity. For Trp in most proteins, the CT state lies well above the fluorescing state, and consists of a quasi-continuum of vibronic states. The coupling between these is reduced by over an order of magnitude by the Franck-Condon factors. Furthermore, most crossings are during fluctuations that are transient and are quickly reversed. Thus, the question of adiabaticity is not clear. Finally, the insensitivity of the absorption bands of Trp in proteins when compared to $3 \mathrm{MI}$ is similar to that for stacking nucleotides, whose dipole-dipole interactions are arguably in the range of several hundred $\mathrm{cm}^{-1}$, requiring one to conclude that the Förster very weak coupling case (Fermi rule) remains valid.

We were further encouraged to retain the non-adiabatic picture because of certain successes emanating from the use of the new parameters. The large interaction produced a completely new picture because the computed energy gap was increased by $\sim 1 \mathrm{eV}\left(D_{\text {vert }}\right.$ was changed from -4000 to $+4700 \mathrm{~cm}^{-1}$ ). Thus, photoinduced ET from Trp is possible only during those short windows of time when a fluctuation brings the ${ }^{1} \mathrm{~L}_{\mathrm{a}}$ and $\mathrm{CT}$ state transiently close in energy. The larger $V_{e l}$ values paired with larger gaps amplifies the effect of heterogeneity in energy gaps due to different protein conformations, which apparently is the cause of the ubiquitous non-exponential decay of Trp fluorescence in proteins. This subject is further discussed in Section 6.

One of the important findings is - at the short distances pertinent to Trp fluorescence quenching - the coupling element does not decrease exponentially with distance between the electron acceptor amide and the indole ring. In fact, we find that through-bond coupling is extremely strong, and quite dependent on rotamer conformation. The rotamer showing the strongest average coupling is that for which the amide is farthest from the indole ring, but with the intervening bonds in a linear configuration that promotes through-bond transfer (see Figure 1). Despite the four-fold difference in the average coupling for different proteins (predicting a 16-fold ET rate difference), there is virtually no correlation of coupling strength and ET rate (experimental and calculated) as deduced from fluorescence quantum yields. This is because of the critical dependence of the energy gap on the electrostatic environment. This finding is quite different from that supposed in earlier studies that placed most of the rate dependence on the coupling strength.[61-63]

Using time averages of the ab initio coupling and density of states just described for individual proteins (i), the Fermi rule based formula became: 


$$
k_{e t}=4 \pi^{2} \mathrm{c}<V_{e l, i}{ }^{2}><\rho\left(\Delta \mathrm{E}_{\mathrm{vert}}(\mathrm{t})+\mathrm{D}_{\mathrm{vert}}\right)>_{\mathrm{t}},
$$

In our most recent formulation, instantaneous rates were computed at each step of $100 \mathrm{~ns}$ simulations for 11 Trps in 8 proteins, giving

$$
k_{e t}=4 \pi^{2} \mathrm{c} V_{e l^{2}}(t) \rho\left(\Delta \mathrm{E}_{\text {vert }}(\mathrm{t})+\mathrm{D}_{\text {vert }}\right),
$$

where instantaneous values of $V_{\text {ell }}(t)$ were taken from tables of values based on the local conformation.[20,64] In this work, we used the much more efficient Gromacs[65] as the MD engine, and for simplicity we ran the simulations with ground state charges and geometry from the forcefield chosen. This necessitated using $D_{\text {vert }}=+200$ to $+2000 \mathrm{~cm}^{-1}$, depending on the forcefield, because the vertical excited state energies were now much higher than when using the CT geometry.

The method has now been applied to a number of interesting proteins.[48,66-71] Despite what would seem to be continual and significant improvements in the method, agreement with experiment has improved but slightly.

Recently, in an elegant tour de force study of $17 \beta$-hairpin and 13 Trp-cage single-Trp miniproteins, Parson, Anderson and co-workers have successfully predicted Trp fluorescence yields using an approach similar to ours, but with significant extensions and variations.[14] The same group performed the experimental measurements on this impressive array of peptides.

In addition, the scope of the Parson computations was much broader than that of the Callis group. One major difference is that almost all conceivable quenching pathways were used in computing the quantum yields. These included ET from the excited indole ring of the Trp to backbone amides beyond the nearest two, and to protonated side chains of nearby histidine, glutamate, aspartate, tyrosine, and cysteine residues. Ionized tyrosine side chains were found to quench strongly by resonance energy transfer or ET to the excited indole ring. Other differences are: the QM program, the form of the density of states function, use of simulations in both the ${ }^{1} \mathrm{~L}_{\mathrm{a}}$ excited state of the Trp and several CT states, the introduction of an interpolation scheme that switches between non-adiabatic and adiabatic ET mechanism, and inclusion of a self-consistent treatment of induced electric dipoles for the reactant and product states.

Less apparent is that Callis uses a single empirical offset of the CT-La energy gap for fitting, whereas Parson and coworkers explicitly compute the excited and CT state energies in separate simulations. Nevertheless, one may point to several adjustable parameters that are embedded in the Parson work, which were tuned to reach good agreement with experiment. These include 1) the non-adiabatic to adiabatic switching point, 2) the density of states function, which is slightly unphysical in appearance, 3 ) a self-consistent treatment of induced electric dipoles for the reactant and product state, in which the polarizabilities were scaled, and 4) choice of chromophore charge scaling, which affects the energy gaps. There was no comparison given for predictions with and without the induced dipoles. 
In summary, this study is an independent verification of our method, and illustrates the way to a more comprehensive approach to simulating Trp fluorescence quenching. Agreement with experiment is similar to what we have found with the larger proteins.

\section{Dielectric compensation of charge modifications}

From our earliest simulations in proteins, [10] the interplay of electrostatic influence from protein and that from water has been eye-catching. In that first work, our prediction of $\lambda_{\max }$ for the protein Staphylococcal nuclease (STNase) was particularly fascinating because the single Trp was sandwiched by two positive charges from two lysines (K110 and K133), and negative charge from a glutamate (E129) was also nearby. The positive ammonium charges are over the benzene ring in such a way as to create an extremely large red shifted $\lambda_{\max }$ - far greater than experimentally observed. We found, however that water compensated with a large blue shift, bringing the steady state $\lambda_{\max }$ prediction into reasonable agreement with the experiment. This intriguing result led to a collaboration in which ultrafast TDFSS was determined for the unmutated protein [wild-type, (WT)] and the four mutants K133A, K110A, E129A, and K110C, for which the new residues alanine (A) and cysteine (C) are uncharged.[72] In this study, Qiu et

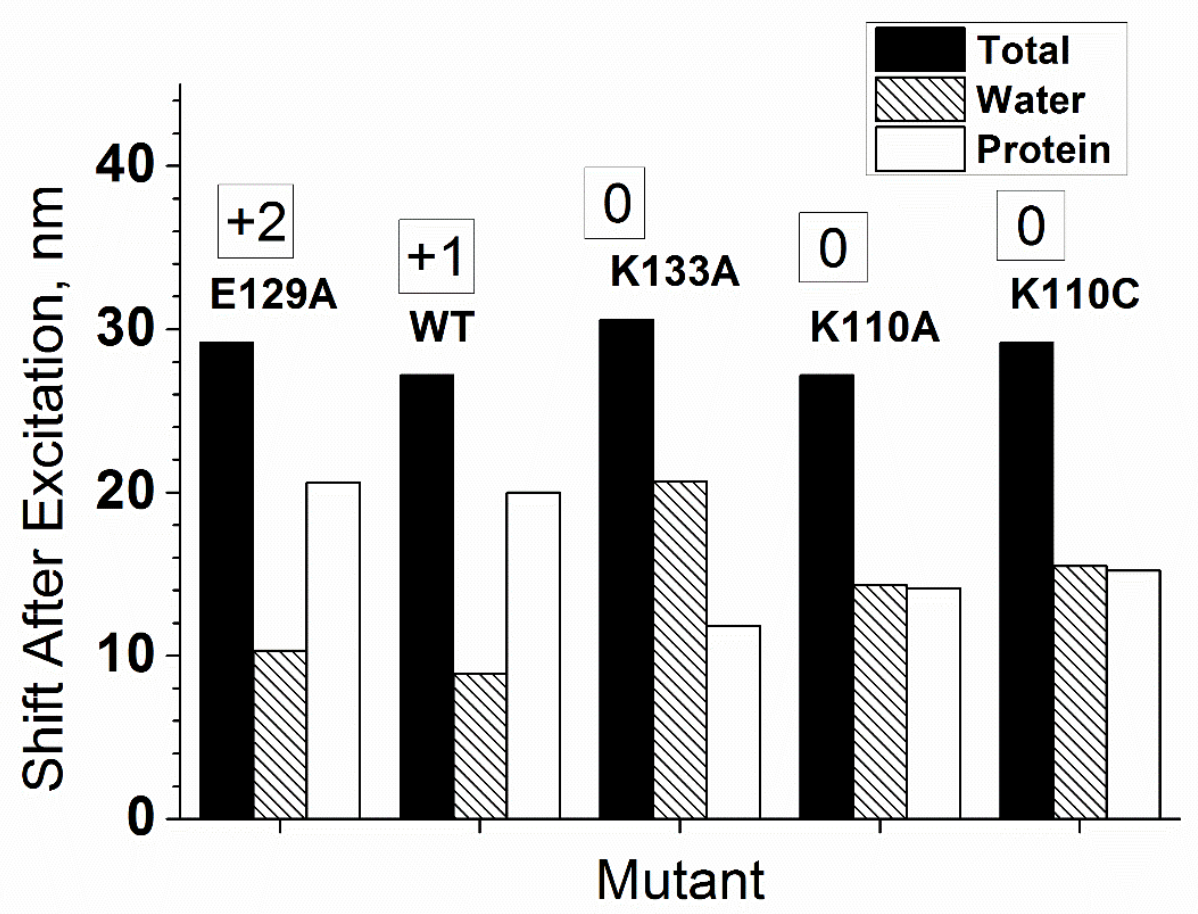

Figure 5. Contributions to the wavelength shift following excitation from protein (white), water (lines), and total of water + protein (black) for Staphylococcal nuclease and the four mutant proteins studied. Also indicated in the boxes are the net charges in close proximity to

al. found a very unexpected result: all five proteins had virtually the same steady state $\lambda_{\max }$ $(\sim 332.5 \pm 0.5 \mathrm{~nm})$. Their conclusion from this extraordinary observation was that the observed Stokes shift is not influenced by the neighboring charged residues, but is dominantly due to hydration. 
This statement is true only as a summary of the net effect. In a recent critique of biological water,[73] Zhong et al. have postulated only one of the possible mechanisms that could explain this surprising behavior, namely that the surrounding protein is too rigid to relax in response to the large, suddenly-produced excited state dipole. But numerous MD simulations $[10,13,66,70,74-81]$ strongly point to a completely different mechanism: the large dielectric compensation by the solvent responding to charged groups that are close to the Trp has a profound effect on the polarization magnitude and direction of water within $15 \AA$ of the Trp. When a charged group that is contributing a large red shift is replaced by a neutral group, the loss of red shift is compensated by a corresponding loss of blue shift from water.

Most recently, we have made an in-depth MD + QM investigation[80] of the STNase charge mutations reported by Qiu et al.[72] The steady state $\lambda_{\max }$ was modeled in both the ground state and in the excited state, each with three sets of 50-ns MD simulations in explicit water. Semiempirical QM and independent electrostatic analysis for the WT protein and the four charge-altering mutants were performed on the chromophore using the coordinates from the simulations. Electrostatic contributions from the nearby charged lysines by themselves contribute 30-90 $\mathrm{nm}$ red shifts relative to the gas phase, but in each case, contributions from water create compensating blue shifts that bring the predicted $\lambda_{\max }$ within $2 \mathrm{~nm}$ of the experimental value, 332 $\pm 0.5 \mathrm{~nm}$ for all five proteins. Although long-range collective interactions from ordered water make large blue shifts, crucial for determining the steady state $\lambda_{\max }$ for absorption and fluorescence, such blue shifts do not contribute to what is measured, the amplitude of the TDFSS following excitation, which we found comes from nearby charges and only about six water molecules tightly networked with those charges. Figure 5 shows the contributions from protein and water to the TDFSS. Here we see that replacing Glu129 with Ala (neutral) has little effect, but replacing Lys 133 causes a marked decrease in average protein contribution, with offsetting increase in water contribution. Replacing Lys 110 with either Ala or Cys results in intermediate contributions. This is reasonable, given that Lys 110 is slightly more distant from Trp140 on average.

It is important to realize that Figure 5 is showing what happens following excitation. At the time of excitation, water is contributing a large blue shift. The magnitude of this blue shift decreases during the relaxation. Water is therefore contributing positively to the time dependent red shift by becoming less blue shifting. In other words, the large excited state dipole on the Trp undoes some of the polarization of the water caused by the protein charges.

Thus, the seemingly unlikely event of mutations at three different sites exactly cancelling the effect of hydration and protein solvation to give nearly the same Stokes shifts and correlation functions, is not simply fortuitous; it is essentially the effect noted and discussed by Halle and Nilsson.[75,79]

Our MD simulations also paint a much different visual picture, contrasting with the structural rigidity suggested by Zhong et al.,[73] wherein the charged groups near Trp in the STNase protein are packed too rigidly to provide a relaxation contribution to the TDFSS. Graphical viewing of the MD trajectories shows, however, a rather fluid nature of the positively charged ammonium groups on the lysines, which are on the end of flexible alkane chains. The flexibility is greatly increased by free rotation of the ammonium group about $\mathrm{C}_{2}-\mathrm{NH}_{3}{ }^{+}$bond, 
where the + charge is carried entirely by the hydrogens. This allows these nearby charges to respond on a time scale of a $\sim 1$ ps to the sudden Coulombic field created by excitation. We are not alone in holding this view.[13,75,79]

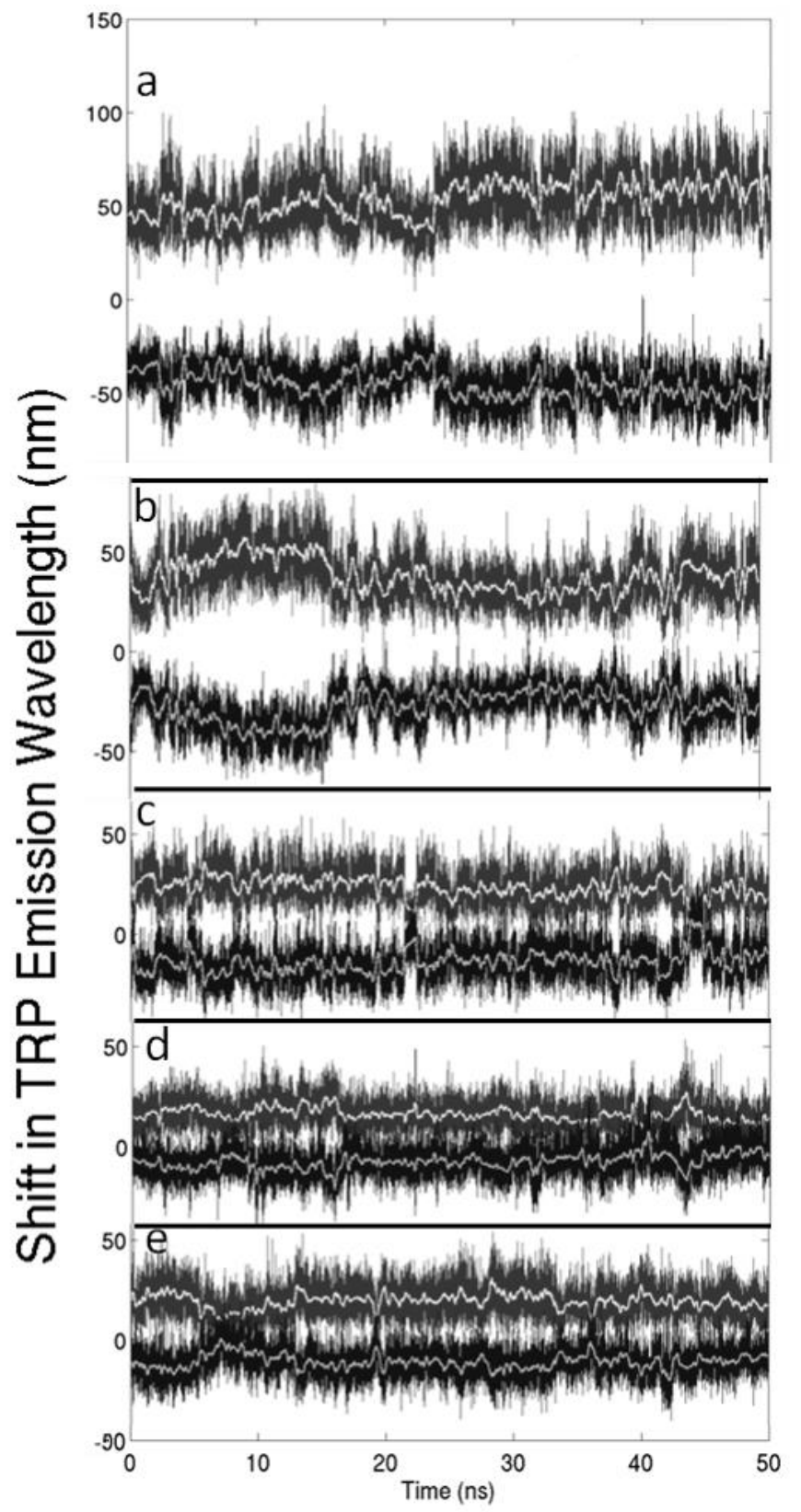

Figure 6. Trajectory of wavelength shift contributions from protein (upper traces) and water + ions (lower traces) for stabilization of ground-- $>{ }^{1} \mathrm{~L}_{\mathrm{a}}$ transition charge differences for WT Staphylococcal nuclease, and charge altering mutants: (a) E129A, (b) WT, (c) K133A, (d) K110A, and (e) K110C. The system is equilibrated to ground state charges during these trajectories. The light traces are smoothed versions of the noisy black traces.

The cancellations of protein and water contributions are also evident in the dynamic fluctuations of instantaneous $\lambda_{\max }$ values, seen in the MD trajectories of Figure 6 . The extremely tight anticorrelation seen especially for WT and E129A has been noted in several studies.[13,70,75,77,79,81-83] This is apparently a direct manifestation of the solvent-protein coupling that is recognized in connection with the 10-100 ps relaxation, [73] even when attributed only to water. The simulations reported here say that both protein and water contribute substantially to the TDFSS, and appear to be strongly linked during fluctuations. We find that protein relaxation contributes considerably to the FSS, and that water contribution to the FSS 
comes only from a few water molecules close to the Trp. These water molecules are part of a tight H-bond network that includes the nearby charges. The motions of the protein and water in this active ensemble are strongly_-yet flexibly_coupled at short time scales, similar to that of "bulk" water. For STNase, the protein charges and water that actually contribute to the FSS are so strongly coupled and correlated near the surface of the protein that we believe whether the slow TDFSS is probing water or protein is not a meaningful question for this protein.

In a closely related study of the single Trp in the small protein GB1,[81] whose structure is known to be unchanged in the $\mathrm{pH}$ range 7 to 3, negative charge surrounding Trp43 was removed by lowering the $\mathrm{pH}$, with virtually the same general result as found by mutating charges in the case of STNase, i.e. that $\lambda_{\max }$ was again invariant within $1 \mathrm{~nm}$. In particular, upon the loss of a negative charge on Glu27 in contact with Trp43, MD simulations showed an increased red shift from protein by $3000 \mathrm{~cm}^{-1}$, with a compensating water contribution changing from a $1000 \mathrm{~cm}^{-1}$ red shift to a $1000 \mathrm{~cm}^{-1}$ blue shift. In common with STNase, Trp43 has a lysine lying above the indole ring, but in GB1 it lies above the pyrrole ring, instead of being over the benzene ring. This creates a blue shift. As with STNase, there is evidence for inseparability of water and protein motions that affect $\operatorname{Trp} \lambda_{\text {max }}$. This strong coupling, however, does not imply rigid; the simulations make clear that coupling is both strong and flexible, and that the previously labeled "bulk water" transient actually carries useful information about the protein-water interface.

As for STNase, pronounced anticorrelation is observed in the contributions of protein and water to both steady state $\lambda_{\max }$ and to the corresponding large fluctuations.

\section{Multiple, discrete, long-lived conformations in proteins}

In 2010, a tremendous leap forward in simulations of protein folding was accomplished by D.E. Shaw and coworkers. [84] A 1-millisecond MD simulation-2 orders of magnitude longer than any previous protein simulation - of bovine pancreatic trypsin inhibitor revealed what had been increasingly inferred from experimental studies: that functional proteins exhibit "basin hopping", i.e. transitions among multiple distinct structural states. These transitions were found to occur on the 10-100 $\mu$ s time scales, typically involving backbone conformational changes, with a large separation of time scales from local side chain dynamics, which occur on the nanosecond scale.

The population of multiple basins ("species") by the ground state ensemble of a protein has for some time been widely believed to be the general origin of the ubiquitous non-exponential decay of Trp fluorescence when the basin hopping rate is on the order of the excited state lifetime, or slower. The idea that these basins are confined only to different rotamer states of Trp[53,85-87] has attained almost dogma status, albeit primarily because of the absence of easily grasped alternatives.

One difficulty with our original ( $a d h o c$ ) choice of $V_{e l}=10 \mathrm{~cm}^{-1}$ with artificially reduced energy gaps, was that heterogeneity of fluorescence lifetimes did not emerge naturally. The most striking challenge was the revealing fluorescence lifetime/NMR study by Barkley and coworkers of a set of seven Trp-containing hexapeptides whose sequences contained no quenching sidechains.[77,88,89] The fluorescence decay curves uniformly exhibited three decay components, whose decay associated spectra showed the common (but not understood) 
correlation that the short wavelength components were associated with the short lifetimes with near perfect correlation. This behavior had emerged in a multitude of similar studies on proteins from many laboratories. Such correlation was not evident in our attempts at simulations of fluorescence wavelengths and lifetimes of the $42(6 \times 7)$ distinct rotomers while using $V_{e l}=10$ $\mathrm{cm}^{-1}$. Using a constant $V_{e l}=200 \mathrm{~cm}^{-1}$ and larger energy gaps $\left(D_{\text {vert }}=+6500 \mathrm{~cm}^{-1}\right)$, however, provided a believable understanding[70] that captured virtually all of the unexpected diversity of the peptides. The study revealed that rotamers having blue-shifted emission spectra, i.e., higher average energy, have an increased probability for quenching, i.e., shorter average lifetime, during large fluctuations in environment that bring the nonfluorescent CT state and the fluorescing state into resonance. Similar success emerged when simulations with these parameters were applied to Trps 68 and156 in human $\gamma \mathrm{D}$ - and Trp72 in human $\gamma \mathrm{S}$-crystallin, in which heterogeneous decay times from 50 to $500 \mathrm{ps}$ were predicted, in agreement with the experiment.[69] These Trps are buried, but water can reach amide oxygen and ring HE1 atoms through narrow channels, greatly stabilizing the amide CT states.

To search for direct evidence for heterogeneity of fluorescence lifetimes in proteins, we have recently carried out 100-ns MD + QM simulations of Trp fluorescence quenching by ET for 20 Trps in 18 proteins[20,64] using the AMBER99SB-ILDN force field[84], and survival curves generated using Equation (4). Average survival curves computed from some of these simulations indeed exhibit non-exponential decay, with some improvement in prediction accuracy for quantum yields as well. Although non-exponential decay has become nearly synonymous with the existence of discrete Trp rotamer states, we found that only 4 of the 20 Trps displayed rotamer transitions during the $100 \mathrm{~ns}$ trajectories. Three of these could be linked to lifetime heterogeneity. Some proteins, however, display considerable heterogeneity with predicted nonexponential decay with no rotamer transitions at all during $100 \mathrm{~ns}$.

We found only slight improvement using these long simulations combined with survival curve analysis, but enough to suggest that that further improvement may result from even longer simulations. The possibility exists, however, that in some cases the interconversion rate between important conformations will be so slow that milliseconds or longer would be required to capture the correct picture.

While these trajectories did not result in significantly more accurate predictions, they are sufficiently long to provide a view of the diversity of protein behavior, which will impact many different protein properties. We feel, however, that we have not explored the question of adiabatic behavior sufficiently to rule out that as a cause for the persistent deviations between experiment and computation.

\section{Contribution to protein folding experiments}

Tryptophan (Trp) fluorescence intensity is commonly used to monitor fast protein folding kinetics. Perhaps most studied is the villin headpiece mutant HP35 (N27H), which has a histidine placed adjacent to the single Trp in a helix, such that at $\mathrm{pH} 5$ the Trp fluorescence is quenched by ET to the histidine cation. This construct was developed by Eaton and coworkers, who measured the folding rate constant by temperature jump experiments[90-92] following an increase in Trp fluorescence (due to a shift in equilibrium to a more unfolded state). 
Recently, however, MD simulations of the folding of this peptide have uniformly indicated that helix III (the location of the Trp-His pair) forms much faster than the measured folding rate, and that the simulated folding rate was considerably longer than the measured value. $[93,94]$ Both results suggested that the experiments could be underestimating the folding rate because the Trp probe is on the fast folding helix III. Independent experiments using an entirely different probe not on helix III, however, confirmed the Trp-His experiments.[92,95] The question became, how could the Trp in the early-folding helix III report the global folding rate?

To help answer this question, we used 1- $\mu$ s MD simulations of HP35 (N27H) and a fasterfolding variant in its folded form at $300 \mathrm{~K}$ to simulate fluorescence quenching caused by ET to the local amide and to the $\mathrm{His}^{+}$. Figure $7 \mathrm{a}$, shows a representative trajectory of the distance between the Trp23 and $\mathrm{His}^{+} 27$ centers of mass (COM). This distance has been considered the best indicator of the quenching rate of Trp fluorescence by His ${ }^{+} .[93,94]$ The important aspect of this and other trajectories are the regions of discrete inter-residue distance. Most striking and significant is the small percentage of time for which the closest distance $(\sim 4 \AA)$ is visited during these trajectories of folded villin - while this region is helical $100 \%$ of the time. In all cases, we

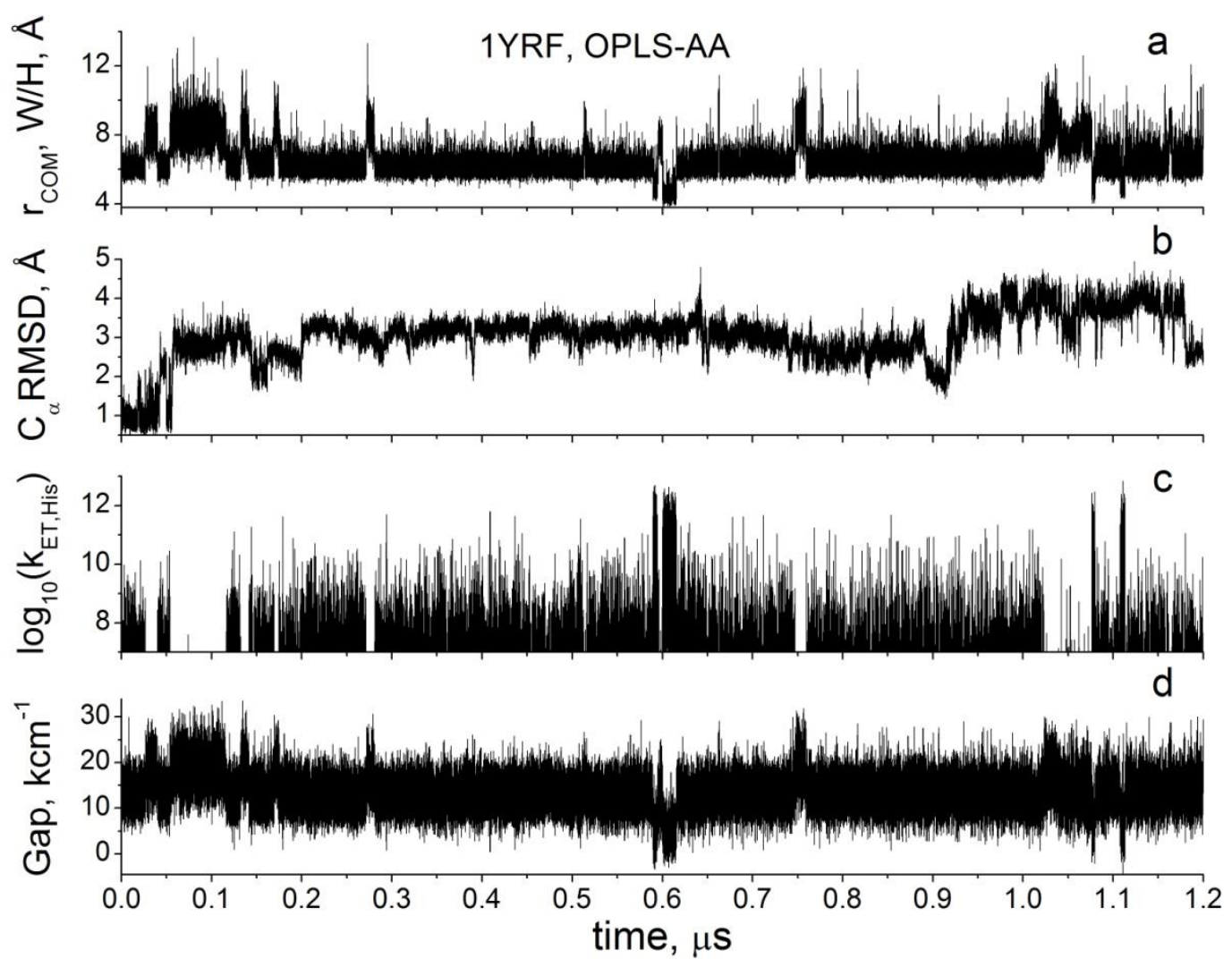

Figure 7. A 1- $\mu$ s simulation of the villin headpiece fragment, $\mathrm{HP} 35\left(\mathrm{~N} 27 \mathrm{H}^{+}\right)$, in explicit water with the OPLS-AA force field. (a) Center of mass (COM) distance between Trp and His $^{+}$rings, (b) $\mathrm{C}_{\alpha}$ RMSD relative to crystal structure (1YRF), (c) calculated ET rate, $\mathrm{s}^{-1}$, for quenching by $\mathrm{His}^{+}$, and (d) $\mathrm{CT}\left(\mathrm{His}^{+}\right)-{ }^{1} \mathrm{~L}_{\mathrm{a}}$ energy gap from $\mathrm{MD}+\mathrm{QM}$. Note the precise correlation between distance, rate, and energy gap, whereas there is no correlation between RMSD and quenching ability for $\mathrm{His}^{+}$. In this simulation, quenching occurs only during the 
find that the closer distance state arises solely through a concerted twisting about two adjacent torsions connecting the Trp ring to the protein backbone (Figure 1). These may be visualized in Figure 8, in which the left most image depicts the x-ray structure, while the center image depicts the quenched state ("close conformation").

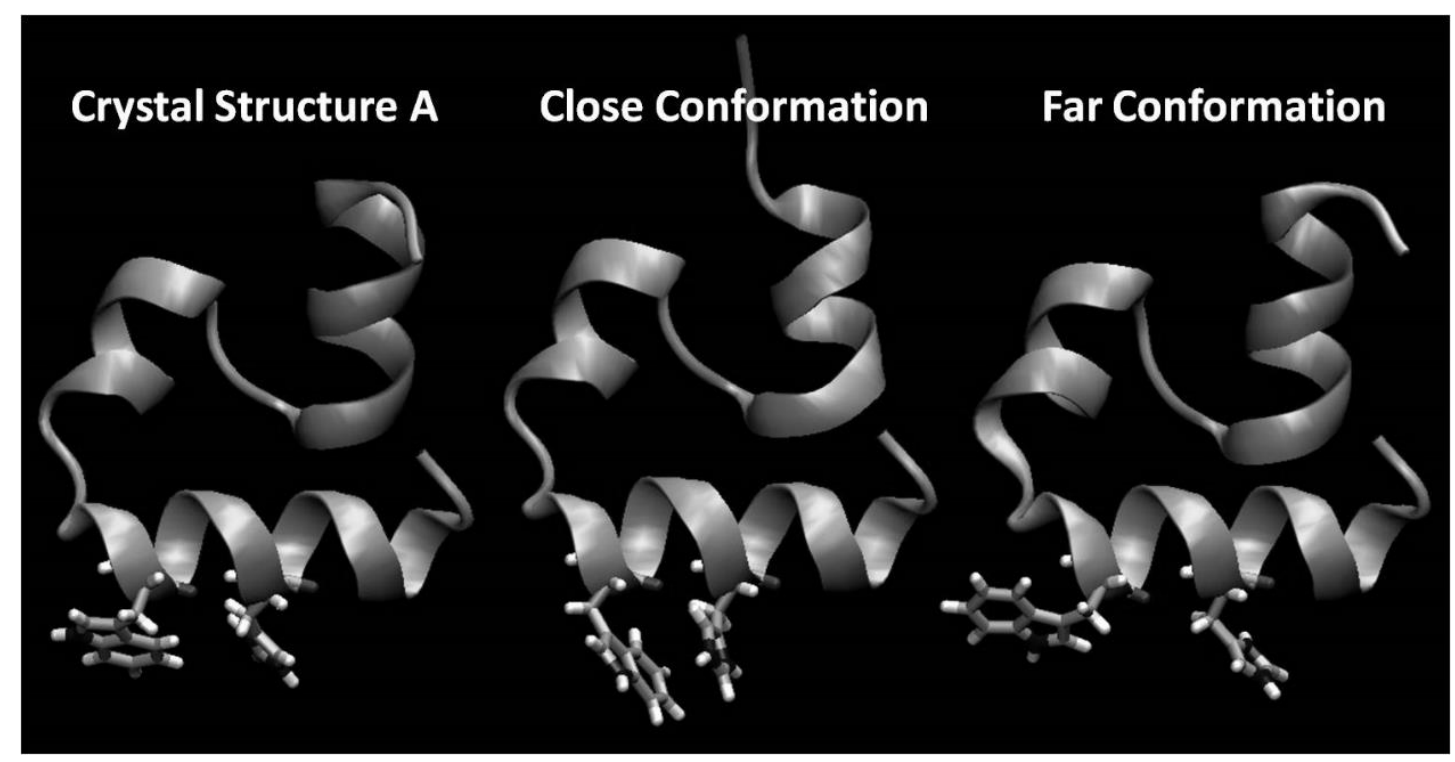

Figure 8. Comparison of the X-ray structure (1YRF "A") with the representative "close" and "far" conformations showing Trp23 and His27 relative conformations taken from a 1- $\mu$ s MD simulation of villin HP35(N27H') at $300 \mathrm{~K}$ in explicit TIP3P water using the AMBER99SB-ILDN forcefield. The X-ray structure on the left, and the structure on the right are the two main "far" conformations, neither of which we believe allows significant quenching. The "close" (center) conformation is reached only transiently for a specific rotamer, and is the only conformation for which we believe quenching is possible. Most of the trajectory is spent in the X-ray conformation, for which we believe quenching is not possible, or is minor.

Figure 7(b) shows the root mean square deviations (RMSD) relative to the crystal structure. The large deviation is because helix III is unraveling during the simulation, a characteristic of the OPLS forcefield. The region including Trp23-His27, however, remains helical, and there is no correlation of the RMSD with the important variables shown in panels Figure 7(a), (c), and (d), all of which exhibit precise correlation.

Most relevant, Figure 7(c) shows that the computed quenching rate constant varies with time in close correlation with the COM of panel Figure 7(a). Figure 7(d) shows the reason: the important energy gap between the CT and ${ }^{1} \mathrm{~L}_{\mathrm{a}}$ states also has an unexpected precise correlation with Trp-His ${ }^{+}$distance. This turned out to be because the CT state formed by ET to His ${ }^{+}$is initially greatly destabilized by strong association with water $\mathrm{O}$ atoms strongly solvating the $\mathrm{His}^{+}$. Such destabilization is much reduced when the Trp and $\mathrm{His}^{+}$are stacked so as to exclude water. Quenching is therefore not possible at longer distances because, as seen in Figure 7d, the large energy gap between the $\mathrm{CT}$ and ${ }^{1} \mathrm{~L}_{\mathrm{a}}$ states means the CT state is out of resonance with the fluorescing state. (For reference, if the quenching rate drops below $1 \times 10^{7} \mathrm{~s}^{-1}$, there is negligible effect on the quantum yield.) We have found, slightly unexpectedly, much the same frequency of 
occurrence of the short distance for a simulation of completely unfolded villin at $400 \mathrm{~K},[71]$ accounting perhaps for the lack of larger increase in fluorescence observed for the unfolding of villin.

Figure 7, therefore, reveals a picture with unexpected details that contrast with the current assumption by experimentalists and computationalists alike: that when the region encompassing these two residues is helical, there will be significant quenching, but there will be no quenching otherwise. This bears directly on the question at hand: how can the Trp in the early folding of helix III report the global folding rate? The key is in the small fraction of time that quenching happens in the helical state. If the equilibrium fraction of quenching state is only $10 \%$, then the Gibbs free energy is only $\sim 1.4 \mathrm{kcal} / \mathrm{mol}$ higher for the quenching state than the non-quenching state. This means that if intermediate unfolded structures, with helix III fully intact, stabilize the quenching state by as little as $2.8 \mathrm{kcal} / \mathrm{mol}$, the quenching rate would increase by a factor of 100 , thus providing a reasonable mechanism by which Trp fluorescence should be able to reliably report the global folding rate. In addition, the fast (70 ns) relaxation time subcomponent reported by Kubelka et al.[91] is consistent with the rate of exchange between the close and far conformations in our simulations, provided we include a temperature dependence of the equilibrium between the close and far conformations of the helical structure arising from a small negative $\Delta \mathrm{H}^{0}$ associated with forming the close conformation.

In summary, these simulations of the villin headpiece demonstrate that quenching by $\mathrm{His}^{+}$in the fully formed helix III is possible only during certain minor $(\sim 10 \%)$ Trp and $\mathrm{His}^{+}$rotamer and solvent conformations. In other words, the quenching rate is quite reasonably expected to be a sensitive function of variable environmental forces on the helix as the protein finds its way in a rugged landscape towards the folded state. This allows the Trp fluorescence on an early-folding helix to report the global folding rate, as recent experiments imply.

\section{Acknowledgements}

We gratefully acknowledge the support from the NSF Grants MCB-0446542 and MCB-0847047, and support from the Extreme Science and Engineering Discovery Environment (XSEDE), which is supported by the National Science Foundation Grant No. OCI-1053575 through Project No. MCB090176.

Students and associates who contributed to the work reported here include Bruce Anderson, Richard Jones, David Theiste, Aden Rehms, Pedro Muino, Lee Slater, David Sammath, David Hahn, Kurt Short, James Vivian, Tiqing Liu, Alexander Petrenko, J. Nathan Scott, Ramon Tusell, Carl Fahlstrom, and Ryan Hutcheson.

The author is also extremely indebted to Mickey Schurr, Bruce Hudson, Dan Harris, Andy Albrecht, Tom Scott, Mary Barkley, Bob Woody, Lennie Brand, Dmitry Toptygin, Lee Spangler, Jaap Broos, Sandy Ross, Jay Knutson, Franck Prendergast, Predrag Ilich, Dongping Zhong, Jan Kubelka, and Bill Eaton for helpful interactions. 


\section{References}

[1] S. Pistolesi, A. Sinicropi, R. Pogni, R. Basosi, N. Ferre, M. Olivucci, Modeling the Fluorescence of Protein-Embedded Tryptophans with ab Initio Multiconfigurational Quantum Chemistry: The Limiting Cases of Parvalbumin and Monellin, J. Phys. Chem. B 113 (2009), p. 16082-16090.

[2] F. Melaccio, N. Ferre, M. Olivucci, Quantum chemical modeling of rhodopsin mutants displaying switchable colors, Phys. Chem. Chem. Phys. 14 (2012), p. 1248512495.

[3] M.M. Huntress, S. Gozem, K.R. Malley, A.E. Jailaubekov, C. Vasileiou, M. Vengris, J.H. Geiger, B. Borhan, I. Schapiro, D.S. Larsen, M. Olivucci, Toward an Understanding of the Retinal Chromophore in Rhodopsin Mimics, J. Phys. Chem. B 117 (2013), p. 10053-10070.

[4] C. Bernini, T. Andruniow, M. Olivucci, R. Pogni, R. Basosi, A. Sinicropi, Effects of the Protein Environment on the Spectral Properties of Tryptophan Radicals in Pseudomonas aeruginosa Azurin, J. Am. Chem. Soc. 135 (2013), p. 4822-4833.

[5] X. Li, L.W. Chung, H. Mizuno, A. Miyawaki, K. Morokuma, Primary Events of Photodynamics in Reversible Photoswitching Fluorescent Protein Dronpa, J. Phys,Chem. Letters 1 (2010), p. 3328-3333.

[6] A. Warshel, Dynamics of reactions in polar solvents. Semiclassical trajectory studies of electron transfer and proton-transfer reactions., J. Phys. Chem. 86 (1982), p. 2218-2224.

[7] S.C.L. Kamerlin, M. Haranczyk, A. Warshel, Progress in Ab Initio QM/MM FreeEnergy Simulations of Electrostatic Energies in Proteins: Accelerated QM/MM Studies of pK(a), Redox Reactions and Solvation Free Energies, J. Phys. Chem. B 113 (2009), p. 1253-1272.

[8] M. Marchi, J.N. Gehlen, D. Chandler, M. Newton, Diabatic surfaces and the pathway for primary electron transfer in a photosynthetic reaction center, J. Am. Chem. Soc. 115 (1993), p. 4178-4190.

[9] J.N. Gehlen, M. Marchi, D. Chandler, Dynamics affecting the primary charge transfer in photosynthesis, Science 263 (1994), p. 499-502.

[10] J.T. Vivian, P.R. Callis, Mechanisms of tryptophan fluorescence shifts in proteins, Biophys. J. 80 (2001), p. 2093-2109.

[11] P.R. Callis, T. Liu, Quantitative prediction of fluorescence quantum yields for tryptophan in proteins, J. Phys. Chem. B 108 (2004), p. 4248-4259. 
[12] D. Toptygin, T.B. Woolf, L. Brand, Picosecond Protein Dynamics: The Origin of the Time-Dependent Spectral Shift in the Fluorescence of the Single Trp in the Protein GB1, J. Phys. Chem. B 114 (2010), p. 11323-11337.

[13] T.P. Li, A.A. Hassanali, S.J. Singer, Origin of Slow Relaxation Following Photoexcitation of W7 in Myoglobin and the Dynamics of Its Hydration Layer, J. Phys. Chem. B 112 (2008), p. 16121-16134.

[14] A.W. McMillan, B.L. Kier, I. Shu, A. Byrne, N.H. Andersen, W.W. Parson, Fluorescence of Tryptophan in Designed Hairpin and Trp-Cage Miniproteins: Measurements of Fluorescence Yields and Calculations by Quantum Mechanical Molecular Dynamics Simulations, J. Phys. Chem. B 117 (2013), p. 1790-1809.

[15] A.Warshel, Computer modeling of chemical reactions in enzymes and solutions (Wiley-Interscience, New York, NY, 1991).

[16] S.C.L. Kamerlin, A. Warshel, At the dawn of the 21st century: Is dynamics the missing link for understanding enzyme catalysis?, Proteins: Struct. , Funct. , Bioinf. 78 (2010), p. 1339-1375.

[17] P.R. Callis, in: Exploring the electrostatic landscape of proteins with tryptophan fluorescence, in Reviews in Fluorescence 2007, ed. C.D.Geddes (Springer, NY, 2009) p. 199.

[18] P.R. Callis, ${ }^{1} L_{a}$ and ${ }^{1} L_{b}$ transitions of tryptophan: Applications of theory and experimental observations to fluorescence of proteins, Methods Enzymol. 278 (1997), p. 113-150.

[19] P.R. Callis, Predicting Fluorescence Lifetimes and Spectra of Biopolymers, Methods Enzymol. (2011);487:1-38.

[20] P.R. Callis. and J.R. Tusell, in: $M D+Q M$ Correlations with Tryptophan Fluorescence Spectral Shifts and Lifetimes, in Fluorescence Spectroscopy and Microscopy, eds. Y.Engelborghs and A.J.W.G.Visser (Springer, Clifton, N.J., 2014) p. 171.

[21] P.R. Callis, Binding Phenomena and Fluorescence Quenching. I: Descriptive Quantum Principles of Fluorescence Quenching using a Supermolecule Approach, J. Mol. Struct. 1077 (2014), p.14-21.

[22] P.R. Callis, Binding Phenomena and Fluorescence Quenching. II: Photophysics of Aromatic Residues and Dependence of Fluorescence Spectra on Protein Conformation, J. Mol. Struct. 1077 (2014), p.22-29.

[23] M. Maroncelli, G.R. Fleming, Computer-Simulation of the Dynamics of Aqueous Solvation, J. Chem. Phys. 89 (1988), p. 5044-5069. 
[24] M. Maroncelli, The dynamics of solvation in polar liquids, J. Mol. Liquids 57 (1993), p. 1-37.

[25] M. Maroncelli, Computer-Simulations of Solvation Dynamics in Acetonitrile, J. Chem. Phys. 94 (1991), p. 2084-2103.

[26] M. Rao, B.J. Berne, Molecular Dynamic Simulation of the Structure of Water in the Vicinity of A Solvated Ion, J. Phys. Chem. 85 (1981), p. 1498-1505.

[27] R. Jimenez, G.R. Fleming, P.V. Kumar, M. Maroncelli, Femtosecond solvation dynamics of water, Nature 369 (1994), p. 471-473.

[28] P.R. Callis, Molecular orbital theory of the ${ }^{1} L_{a}$ and ${ }^{1} L_{b}$ states of indole, J. Chem. Phys. 95 (1991), p. 4230-4240.

[29] P. Ilich, C. Haydock, F.G. Prendergast, Electronic-Transitions in Hydrated Indole - A Md Indo S Study, Chem. Phys. Lett. 158 (1989), p. 129-134.

[30] P.L. Muino, D. Harris, J. Berryhill, B. Hudson, P.R. Callis, Simulation of Solvent Dynamics Effects on the Fluorescence of 3- Methylindole in Water, Proc. SPIEInt. Soc. Opt. Eng. 1640 (1992), p. 240-250.

[31] P.L. Muino, P.R. Callis, Hybrid simulations of solvation effects on electronic spectra: indoles in water, J. Chem. Phys. 100 (1994), p. 4093-4109.

[32] J. Ridley, M. Zerner, Intermediate Neglect of Differential Overlap (INDO) Technique for Spectroscopy: Pyrrole and the Azines, Theor. Chim. Acta(Berl) 32 (1973), p. 111-134.

[33] M.A. Thompson, M.C. Zerner, A theoretical examination of the electronic structure and spectroscopy of the photosynthetic reaction center from Rhodopseudomonas viridis, J. Am. Chem. Soc. 113 (1991), p. 8210-8215.

[34] M.G. Cory, M.C. Zerner, X.C. Xu, K. Schulten, Electronic excitations in aggregates of bacteriochlorophylls, Journal of Physical Chemistry B 102 (1998), p. 76407650 .

[35] G. Segal, Pople,John - the CNDO and INDO Methods, J. Phys. Chem. 94 (1990), p. 5436-5439.

[36] D. Theiste, P.R. Callis, R.W. Woody, Effects of the crystal field on transition moments in 9- ethylguanine, J. Am. Chem. Soc. 113 (1991), p. 3260-3267.

[37] N. Sreerama, R.W. Woody, P.R. Callis, Theoretical study of the crystal field effects on the transition dipole moments in methylated adenines, J. Phys. Chem. 98 (1994), p. 10397-10407. 
[38] D.M. Sammeth, S.S. Siewert, L.H. Spangler, P.R. Callis, ${ }^{1} L_{a}$ transitions of jet-cooled 3-methylindole, Chem. Phys. Lett. 193 (1992), p. 532-538.

[39] P.R. Callis, A.A. Rehms, Two-photon fluorescence excitation spectra of aromatic amino acids, Chem. Phys. Lett. 208 (1993), p. 276-282.

[40] B.J. Fender, D.M. Sammeth, P.R. Callis, Site selective photoselection study of indole in argon matrix: location of the ${ }^{1} L_{a}$ orgin, Chem. Phys. Lett. 239 (1995), p. 31-37.

[41] B.J. Fender, P.R. Callis, ${ }^{1} L_{a}$ origin Locations of methylindoles in argon matrices, Chem. Phys. Lett. 262 (1996), p. 343-348.

[42] E.A. Burstein, S.M. Abornev, Y.K. Reshetnyak, Decomposition of protein tryptophan fluorescence spectra into log-normal components. I. Decomposition algorithms, Biophys. J. 81 (2001), p. 1699-1709.

[43] Y.K. Reshetnyak, E.A. Burstein, Decomposition of protein tryptophan fluorescence spectra into log-normal components. II. The statistical proof of discreteness of tryptophan classes in proteins, Biophys. J. 81 (2001), p. 1710-1734.

[44] Y.K. Reshetnyak, Y. Koshevnik, E.A. Burstein, Decomposition of protein tryptophan fluorescence spectra into log-normal components. III. Correlation between fluorescence and microenvironment parameters of individual tryptophan residues, Biophys. J. 81 (2001), p. 1735-1758.

[45] T. Takigawa, T. Ashida, Y. Sasada, M. Kakudo, Crystal Structures of L-Tryptophan Hydrochloride and Hydrobromide, Bull. Chem. Soc. Jpn. 39 (1966), p. 23692378.

[46] J. Broos, K. Tveen-Jensen, E. de Waal, B.H. Hesp, J.B. Jackson, G.W. Canters, P.R. Callis, The emitting state of tryptophan in proteins with highly blue-shifted fluorescence, Angew. Chem. Int. Ed. Engl. 46 (2007), p. 5137-5139.

[47] K.T. Jensen, G. Strambini, G. Gonnelli, J. Broos, J.B. Jackson, Mutations in transhydrogenase change the fluorescence emission state of trp-72 from ${ }^{1} L_{a}$ to ${ }^{1} L_{b}$, Biophys. J 95 (2008), p. 3419-3428.

[48] P.R. Callis, T.Q. Liu, Short range photoinduced electron transfer in proteins: QM-MM simulations of tryptophan and flavin fluorescence quenching in proteins, Chem. Phys. 326 (2006), p. 230-239.

[49] S.R. Meech, A. Lee, D. Phillips, On the nature of the fluorescent state of methylated indole derivatives, Chem. Phys. 80 (1983), p. 317-328.

[50] R.W. Cowgill, Fluorescence and the structure of proteins. I. Effects of substituents on the fluorescence of indole and phenol compounds, Arch. Biochm. Biophys. 100 (1963), p. 36-44. 
[51] J. Feitelson, Environmental effects on the fluorescence of tryptophan and other indole derivatives, Israel J. Chem. 8 (1970), p. 241-252.

[52] R.W. Ricci, J.M. Nesta, Inter- and intramolecular quenching of indole fluorescence by carbonyl compounds, J. Phys. Chem. 80 (1976), p. 974-980.

[53] J.W. Petrich, M.C. Chang, D.B. McDonald, G.R. Fleming, On the origin of nonexponential fluorescence decay in tryptophan and its derivatives, J. Am. Chem. Soc. 105 (1983), p. 3824-3832.

[54] J. Li, B. Williams, C.J. Cramer, D.G. Truhlar, A class IV charge model for molecular excited states, J. Phys. Chem. 110 (1999), p. 724-733.

[55] N.R. Kestner, J. Logan, J. Jortner, Thermal electron transfer reactions in polar solvents, J. Phys. Chem. 78 (1974), p. 2148-2166.

[56] M. Bixon, J. Jortner, Electron transfer--From isolated molecules to biomolecules, Adv. Chem. Phys. 106 (1999), p. 35-202.

[57] M. Tachiya, Generalization of the Marcus equation for the electron-transfer rate, J. Phys. Chem. 97 (1993), p. 5911-5916.

[58] P.R. Callis, J.T. Vivian, Understanding the variable fluorescence quantum yield of tryptophan in proteins using QM-MM simulations. Quenching by charge transfer to the peptide backbone, Chem. Phys. Lett. 369 (2003), p. 409-414.

[59] P.R. Callis, J.T. Vivian, L.S. Slater, Ab initio calculations of vibronic spectra for indole, Chem. Phys. Lett. 244 (1995), p. 53-58.

[60] P.R. Callis, A. Petrenko, P.L. Muino, J.R. Tusell, Ab initio prediction of tryptophan fluorescence quenching by protein electric field enabled electron transfer, J. Phys. Chem. B 111 (2007), p. 10335-10339.

[61] A. Sillen, J. Hennecke, D. Roethlisberger, R. Glockshuber, Y. Engelborghs, Fluorescence quenching in the DsbA protein from Escherichia coli: The complete picture of the excited-state energy pathway and evidence for the reshuffling dynamics of the microstates of tryptophan, Proteins 37 (1999), p. 253-263.

[62] A. Sillen, J.F. Diaz, Y. Engelborghs, A step toward the prediction of the fluorescence lifetimes of tryptophan residues in proteins based on structural and spectral data, Protein Sci. 9 (2000), p. 158-169.

[63] M. Hellings, M. DeMaeyer, S. Verheyden, Q. Hao, E.J.M. VanDamme, W.J. Peumans, Y. Engelborghs, The Dead-End elimination method, tryptophan rotamers, and fluorescence lifetimes, Biophys. J. 85 (2003), p. 1894-1902. 
[64] Tusell, J. R.,Computation of fluorescence quenching by amide and histidine, Ph.D. Dissertation, Montana State University (2011)

[65] B. Hess, C. Kutzner, D. van der Spoel, E. Lindahl, Algorithms for Highly Efficient, Load-Balanced, and Scalable Molecular Simulation, J. Chem. Theory Comp. 4 (2008), p. 435-447.

[66] L.C. Kurz, B. Fite, J. Jean, J. Park, T. Erpelding, P. Callis, Photophysics of tryptophan fluorescence: Link with the catalytic strategy of the citrate synthase from Thermoplasma acidophilum, Biochemistry 44 (2005), p. 1394-1413.

[67] J.H. Xu, D. Toptygin, K.J. Graver, R.A. Albertini, R.S. Savtchenko, N.D. Meadow, S. Roseman, P.R. Callis, L. Brand, J.R. Knutson, Ultrafast fluorescence dynamics of tryptophan in the proteins monellin and IIA(Glc), J. Am. Chem. Soc. 128 (2006), p. 1214-1221.

[68] J. Chen, P.R. Callis, J. King, Mechanism of the Very Efficient Quenching of Tryptophan Fluorescence in Human gamma D-and gamma S-Crystallins: The gamma-Crystallin Fold May Have Evolved To Protect Tryptophan Residues from Ultraviolet Photodamage, Biochemistry 48 (2009), p. 3708-3716.

[69] J.H. Xu, J.J. Chen, D. Toptygin, O. Tcherkasskaya, P. Callis, J. King, L. Brand, J.R. Knutson, Femtosecond Fluorescence Spectra of Tryptophan in Human gammaCrystallin Mutants: Site-Dependent Ultrafast Quenching, J. Am. Chem. Soc. 131 (2009), p. 16751-16757.

[70] C.P. Pan, P.L. Muino, M.D. Barkley, P.R. Callis, Correlation of Tryptophan Fluorescence Spectral Shifts and Lifetimes Arising Directly from Heterogeneous Environment, J. Phys. Chem. B 115 (2011), p. 3245-3253.

[71] J.R. Tusell, P.R. Callis, Simulations of Tryptophan Fluorescence Dynamics during Folding of the Villin Headpiece, J. Phys. Chem. B 116 (2012), p. 2586-2594.

[72] W.H. Qiu, Y.T. Kao, L.Y. Zhang, Y. Yang, L.J. Wang, W.E. Stites, D.P. Zhong, A.H. Zewail, Protein surface hydration mapped by site-specific mutations, Proc. Natl. Acad. Sci. U. S. A. 103 (2006), p. 13979-13984.

[73] D.P. Zhong, S.K. Pal, A.H. Zewail, Biological water: A critique, Chem. Phys,Lett. 503 (2011), p. 1-11.

[74] P.R. Callis, W.R. Kirk, F.G. Prendergast, $p H$-Induced fluorescence shifts in E. coli thioredoxin mutants. Comparison of experiment and theory, Biophys. J. 78 (2000), p. 127A.

[75] L. Nilsson, B. Halle, Molecular origin of time-dependent fluorescence shifts in proteins, Proc. Natl. Acad. Sci. U. S. A. 102 (2005), p. 13867-13872. 
[76] J.J. Chen, S.L. Flaugh, P.R. Callis, J. King, Mechanism of the highly efficient quenching of tryptophan fluorescence in human gamma D-crystallin, Biochemistry 45 (2006), p. 11552-11563.

[77] C.P. Pan, P.R. Callis, M.D. Barkley, Dependence of tryptophan emission wavelength on conformation in cyclic hexapeptides, J. Phys. Chem. B 110 (2006), p. 70097016.

[78] T.P. Li, A.A.P. Hassanali, Y.T. Kao, D.P. Zhong, S.J. Singer, Hydration dynamics and time scales of coupled water-protein fluctuations, J. Am. Chem. Soc. 129 (2007), p. 3376-3382.

[79] B. Halle, L. Nilsson, Does the Dynamic Stokes Shift Report on Slow Protein Hydration Dynamics?, J. Phys. Chem. 113 (2009), p. 8210-8213.

[80] J.N. Scott, P.R. Callis, Insensitivity of Tryptophan Fluorescence to Local Charge Mutations, J. Phys. Chem. B 117 (2013), p. 9598-9605.

[81] A. Biesso, J.H. Xu, P.L. Muiño, P.R. Callis, J.R. Knutson, Charge Invariant ProteinWater Relaxation in GB1 via Ultrafast Tryptophan Fluorescence, J. Am. Chem. Soc. 136 (2014), p. 2739-2747.

[82] D.N. Le Bard, D.V. Matyushov, Ferroelectric Hydration Shells around Proteins: Electrostatics of the Protein-Water Interface, J. Phys. Chem. B 114 (2010), p. 9246-9258.

[83] D.V. Matyushov, Dipolar response of hydrated proteins, J. Chem. Phys. 136 (2012), p. 085102-1-085102-15

[84] D.E. Shaw, P. Maragakis, K. Lindorff-Larsen, S. Piana, R.O. Dror, M.P. Eastwood, J.A. Bank, J.M. Jumper, J.K. Salmon, Y.B. Shan, W. Wriggers, Atomic-Level Characterization of the Structural Dynamics of Proteins, Science 330 (2010), p. 341-346.

[85] A.G. Szabo, D.M. Rayner, Fluorescence Decay of Tryptophan Conformers in Aqueous Solution, J. Am. Chem. Soc. 102 (1980), p. 554-563.

[86] T.E.S. Dahms, K.J. Willis, A.G. Szabo, Conformational heterogeneity of tryptophan in a protein crystal, J. Am. Chem. Soc. 117 (1995), p. 2321-2326.

[87] B. Donzel, P. Gaudechon, P. Wahl, Study of the Conformation in the Excited State of Two Tryptophanyl Diketopiperazines, J. Am. Chem. Soc. 96 (1974), p. 801-808.

[88] P.D. Adams, Y. Chen, K. Ma, M.G. Zagorski, F.D. Sonnichsen, M.L. McLaughlin, M.D. Barkley, Intramolecular Quenching of Tryptophan Fluorescence by the Peptide Bond in Cyclic Hexapeptides, J. Am. Chem. Soc. 124 (2002), p. 92789286. 
[89] C.P. Pan, M.D. Barkley, Conformational effects on tryptophan fluorescence in cyclic hexapeptides, Biophys. J. 86 (2004), p. 3828-3835.

[90] J. Kubelka, E.R. Henry, T. Cellmer, J. Hofrichter, W.A. Eaton, Chemical, physical, and theoretical kinetics of an ultrafast folding protein, Proc. Natl. Acad. Sci. USA 105 (2008), p. 18655-18662.

[91] J. Kubelka, T.K. Chiu, D.R. Davies, W.A. Eaton, J. Hofrichter, Sub-microsecond protein folding, J. Mol. Biol. 359 (2006), p. 546-553.

[92] T. Cellmer, M. Buscaglia, E.R. Henry, J. Hofrichter, W.A. Eaton, Making connections between ultrafast protein folding kinetics and molecular dynamics simulations, Proc. Natl. Acad. Sci. USA 108 (2011), p. 6103-6108.

[93] P.L. Freddolino, K. Schulten, Common Structural Transitions in Explicit-Solvent Simulations of Villin Headpiece Folding, Biophys. J. 97 (2009), p. 2338-2347.

[94] D.L. Ensign, P.M. Kasson, V.S. Pande, Heterogeneity even at the speed limit of folding: Large-scale molecular dynamics study of a fast-folding variant of the villin headpiece, J. Mol. Biol. 374 (2007), p. 806-816.

[95] A. Reiner, P. Henklein, T. Kiefhaber, An unlocking/relocking barrier in conformational fluctuations of villin headpiece subdomain, Proc. Natl. Acad. Sci. USA 107 (2010), p. 4955-4960. 